\title{
A NONLINEAR BOUNDARY PROBLEM
}

\author{
JOHN R. HATCHER
}

\begin{abstract}
A nonlinear Hilbert problem of power type is solved in closed form by representing a sectionally holomorphic function by means of an integral with power kernel. This technique transforms the problem to one of solving an integral equation of the generalized Abel type.
\end{abstract}

1. Introduction. In this paper we consider the problem of finding a function $\Phi(z)=u+i v$, holomorphic in the plane cut along the interval $[0,1]$ and vanishing at infinity, such that

$$
\left[\Phi^{+}(x)\right]^{\alpha}+\left[\Phi^{-}(x)\right]^{\alpha}=f \quad \text { on }(0,1), \quad 0<\alpha<1,
$$

where, as usual, $\Phi^{ \pm}(x)$ are the limiting values of $\Phi(z)$ on the approaches to the cut from above and below, respectively. As basis for the presented process of solution it is assumed that $f(x) \in D(H)$. The class $D(H)$ is the union of all functions $f(x)$ with a derivative satisfying a Hölder condition on $[0,1]$ with the possible exception of the endpoints 0,1 , but, for $x \in(0,1)$ and near $p$,

$$
\left|f^{\prime}(x)\right| \leqslant M /|x-p|^{\mu}
$$

$p$ stands for either of the endpoints, and $M, \mu$ are positive constants, $\mu<1$.

It appears that the nonlinear boundary problem (1.1) is related in some manner to an ordinary linear nonhomogeneous Hilbert problem. However, we avoid this approach to the solution because $\Phi^{\alpha}$ may not be analytic. Our procedure then is to put

$$
\Phi(z)=\left(\int_{0}^{1}(t-z)^{-\alpha} \varphi(t) d t\right)^{1 / \alpha}, \quad z \notin[0,1],
$$

where the function $\varphi$ is sought in the class defined by

$$
\varphi(x)=\frac{\varphi^{*}(x)}{x^{1-\varepsilon_{1}}(1-x)^{1-\varepsilon_{2}}}, \quad 0<x<1,
$$

with $\varepsilon_{1}, \varepsilon_{2}>0$ and $\varphi^{*}(x)$ is Hölder continuous on $[0,1]$.

The limiting values $\Phi^{ \pm}(x)$ of $\Phi(z)$ on the approaches to the cut from above and below will be needed. Consequently, we define

$$
\begin{gathered}
\arg (t-z) \rightarrow \mp \pi, \quad z \rightarrow x \pm i 0, \quad 0 \leqslant t<x \leqslant 1 \\
\arg (t-z) \rightarrow 0, \quad z \rightarrow x \pm i 0, \quad 0 \leqslant x<t \leqslant 1
\end{gathered}
$$

Received by the editors May 4, 1984 and, in revised form, November 21, 1984.

1980 Mathematics Subject Classification. Primary 30E25; Secondary 45G05.

Key words and phrases. Cauchy integral, Plemelj formulae, nonhomogeneous boundary value problem. 
Then it follows from (1.2) that

$$
\Phi^{ \pm}(x)=\left[J_{1 \alpha}(x) e^{ \pm i \pi \alpha}+J_{2 \alpha}(x)\right]^{1 / \alpha},
$$

where

$$
J_{1 \alpha}(x)=\int_{0}^{x}(x-t)^{-\alpha} \varphi(t) d t, \quad J_{2 \alpha}(x)=\int_{x}^{1}(t-x)^{-\alpha} \varphi(t) d t .
$$

Thus, by substituting into (1.1) the limiting values $\Phi^{ \pm}(x)$ from formulae (1.4), we obtain

$$
2 J_{1 \alpha}(x) \cos \pi \alpha+2 J_{2 \alpha}(x)=f(x) .
$$

Equation (1.5) is a special case of the type known as Abel's generalized integral equation [1]. Its solution in the class defined by (1.3) determines the function $\Phi(z)$ given by (1.2).

2. Solution of the generalized Abel equation. Consider the sectionally holomorphic function $\Omega$ defined by

$$
\Omega(z)=[z(1-z)]^{(\alpha-1) / 2} \int_{0}^{1}(t-z)^{-\alpha} \varphi(t) d t, \quad z \notin[0,1],
$$

where some branch of the many-valued function $[z(1-z)]^{(\alpha-1) / 2}(t-z)^{-\alpha}$ is chosen.

Define $\arg z \rightarrow 0$ as $z \rightarrow x+i 0$ and $\arg z \rightarrow 2 \pi$ as $z \rightarrow x-i 0$ for $0<x<1$. Then it follows that

$$
\left\{\begin{array}{l}
R(x) \Omega^{+}(x)=J_{1 \alpha}(x) e^{i \pi \alpha}+J_{2 \alpha}(x) \\
-R(x) \Omega^{-}(x)=J_{1 \alpha}(x)+J_{2 \alpha}(x) e^{i \pi \alpha}
\end{array}\right.
$$

where $R(x)=[x(1-x)]$.

Solving (2.1) for $J_{1 \alpha}(x), J_{2 \alpha}(x)$ and inserting these values into (1.5), we obtain the nonhomogeneous Hilbert problem $\Omega^{+}(x)=e^{-i \pi \alpha} \Omega^{-}(x)+f(x) / R(x)$ or, equivalently,

$$
\frac{\Omega^{+}(x)}{H^{+}(x)}-\frac{\Omega^{-}(x)}{H^{-}(x)}=\frac{f(x)}{R(x) H^{+}(x)},
$$

where $H(z)=z^{\alpha / 2}(1-z)^{-\alpha / 2}$. Thus, we have

$$
\Omega(z)=\frac{H(z)}{2 \pi i} \int_{0}^{1} \frac{f(t) d t}{H^{+}(t) R(t)(t-z)},
$$

so that by the well-known Plemelj formulae [2] for the limiting values of a Cauchy integral, we have

$$
\Omega^{ \pm}(x)=H^{ \pm}(x)\left(\frac{ \pm f(x)}{2 H^{+}(x) R(x)}+\frac{1}{2 \pi i} \int_{0}^{1} \frac{f(x) d t}{H^{+}(t) R(t)(t-x)}\right)
$$


Now, by virtue of (2.1) and (2.2), it follows that

$$
\begin{aligned}
J_{1 \alpha}(x) & =\frac{R(x)\left[\Omega^{+}(x) e^{i \pi \alpha}+\Omega^{-}(x)\right]}{e^{2 \pi i \alpha}-1} \\
& =\frac{-R(x) H^{+}(x)}{2 \pi \sin \pi \alpha} \int_{0}^{1} \frac{f(t) d t}{H^{+}(t) R(t)(t-x)} \\
& =\frac{-\sqrt{x}(1-x)^{1 / 2-\alpha}}{2 \pi \sin \pi \alpha} \int_{0}^{1} \frac{(1-t)^{\alpha} f(t) d t}{\sqrt{t} \sqrt{1-t}(t-x)} .
\end{aligned}
$$

Equation (2.3) is an ordinary Abel equation to be solved for $\varphi$. Because the solution depends on the differentiability of the right-hand side of (2.3), we establish the following lemma.

Lemma. For $f(x) \in D(H)$ and $0<\alpha<1$, let

$$
Q(x)=\sqrt{x} \sqrt{1-x} \int_{0}^{1} \frac{(1-t)^{\alpha} f(t) d t}{\sqrt{t} \sqrt{1-t}(t-x)}, \quad 0<x<1,
$$

where the integral is taken in the sense of principal value. Then $Q(x)$ is differentiable on $0<x<1$ and

$$
Q^{\prime}(x)=\frac{1}{\sqrt{x} \sqrt{1-x}} \int_{0}^{1} \frac{\sqrt{t} \sqrt{1-t}\left[(1-t)^{\alpha} f(t)\right]^{\prime} d t}{t-x} .
$$

Proof. For sufficiently small $\varepsilon>0$ put

$$
I_{\varepsilon}(x)=\int_{0}^{x-\varepsilon} \frac{(1-t)^{\alpha} f(t) d t}{\sqrt{t} \sqrt{1-t}(t-x)}+\int_{x+\varepsilon}^{1} \frac{(1-t)^{\alpha} f(t) d t}{\sqrt{t} \sqrt{1-t}(t-x)} .
$$

Also let

$$
A(x, t)=\log \left|\frac{\sqrt{x} \sqrt{1-t}-\sqrt{t} \sqrt{1-x}}{\sqrt{x} \sqrt{1-t}+\sqrt{t} \sqrt{1-x}}\right|, \quad t \neq x .
$$

Then for fixed $x$ and variable $t$ we have

$$
\frac{d A(x, t)}{\sqrt{x} \sqrt{1-x}}=\frac{d t}{\sqrt{t} \sqrt{1-t}(t-x)},
$$

and it follows from integration by parts that

$\sqrt{x} \sqrt{1-x} I_{\varepsilon}(x)$

$$
\begin{aligned}
= & (1-x+\varepsilon)^{\alpha} f(x-\varepsilon) A(x, x-\varepsilon)-(1-x-\varepsilon)^{\alpha} f(x+\varepsilon) A(x, x+\varepsilon) \\
& -\int_{0}^{x-\varepsilon} A(x, t)\left[(1-t)^{\alpha} f(t)\right]^{\prime} d t-\int_{x+\varepsilon}^{1} A(x, t)\left[(1-t)^{\alpha} f(t)\right]^{\prime} d t .
\end{aligned}
$$

Recall the meaning of $A(x, t)$, and then rationalize and rearrange some terms to find that

$$
\begin{aligned}
(1-x+\varepsilon)^{\alpha} f & (x-\varepsilon) A(x, x-\varepsilon)-(1-x-\varepsilon)^{\alpha} f(x+\varepsilon) A(x, x+\varepsilon) \\
= & 2(1-x-\varepsilon)^{\alpha} f(x+\varepsilon) \log (\sqrt{x} \sqrt{1-x-\varepsilon}+\sqrt{x+\varepsilon} \sqrt{1-x}) \\
& -2(1-x+\varepsilon)^{\alpha} f(x-\varepsilon) \log (\sqrt{x} \sqrt{1-x+\varepsilon}+\sqrt{x-\varepsilon} \sqrt{1-x}) \\
& +\left[(1-x+\varepsilon)^{\alpha} f(x-\varepsilon)-(1-x-\varepsilon)^{\alpha} f(x+\varepsilon)\right] \log \varepsilon .
\end{aligned}
$$


Thus it follows that

$$
\begin{aligned}
Q(x) & =\sqrt{x} \sqrt{1-x} \lim _{\varepsilon \rightarrow 0} I_{\varepsilon}(x) \\
& =-\int_{0}^{1} A(x, t)\left[(1-t)^{\alpha} f(t)\right]^{\prime} d t \\
& =\int_{0}^{1}\left[(1-t)^{\alpha} f(t)\right]^{\prime} \log \left|\frac{\sqrt{x} \sqrt{1-t}+\sqrt{t} \sqrt{1-x}}{\sqrt{x} \sqrt{1-t}-\sqrt{t} \sqrt{1-x}}\right| d t
\end{aligned}
$$

or, equivalently,

$$
\begin{aligned}
Q(x)= & 2 \int_{0}^{1}\left[(1-t)^{\alpha} f(t)\right]^{\prime} \log (\sqrt{x} \sqrt{1-t}+\sqrt{t} \sqrt{1-x}) d t \\
& -\int_{0}^{1}\left[(1-t)^{\alpha} f(t)\right]^{\prime} \log |t-x| d t
\end{aligned}
$$

The second integral in (2.4) is understood in the sense of principal value. Denote this integral by $J(x)$ and put

$$
\begin{aligned}
J_{\varepsilon}(x)= & \int_{0}^{x-\varepsilon}\left[(1-t)^{\alpha} f(t)\right]^{\prime} \log |t-x| d t \\
& +\int_{x+\varepsilon}^{1}\left[(1-t)^{\alpha} f(t)\right]^{\prime} \log |t-x| d t
\end{aligned}
$$

where $\varepsilon>0$ is sufficiently small. Now

$$
\begin{aligned}
J_{\varepsilon}^{\prime}(x)=[ & (1-x+\varepsilon)^{\alpha} f^{\prime}(x-\varepsilon)-(1-x-\varepsilon)^{\alpha} f^{\prime}(x+\varepsilon) \\
& \left.+\alpha(1-x-\varepsilon)^{\alpha-1} f(x+\varepsilon)-\alpha(1-x+\varepsilon)^{\alpha-1} f(x-\varepsilon)\right] \log \varepsilon \\
& -\int_{0}^{x-\varepsilon} \frac{\left[(1-t)^{\alpha} f(t)\right]^{\prime}}{t-x} d t-\int_{x+\varepsilon}^{1} \frac{\left[(1-t)^{\alpha} f(t)\right]^{\prime}}{t-x} d t,
\end{aligned}
$$

and hence $J_{\varepsilon}^{\prime}(x)$ converges uniformly to the limit

$$
\lim _{\varepsilon \rightarrow 0} J_{\varepsilon}^{\prime}(x)=-\int_{0}^{1} \frac{\left[(1-t)^{\alpha} f(t)\right]^{\prime}}{t-x} d t .
$$

Let this limit be denoted by $L(x)$. Now for any $x \in(0,1)$, pick $x_{0}$ such that $0<x_{0}<x$. Thus, since $J_{\varepsilon}^{\prime}(x)$ converges uniformly to $L(x)$, we have

$$
\int_{x_{0}}^{x} L(t) d t=\lim _{\varepsilon \rightarrow 0} \int_{x_{0}}^{x} J_{\varepsilon}^{\prime}(t) d t=J(x)-J\left(x_{0}\right) .
$$

But $L(x)$ is continuous, and therefore $J^{\prime}(x)$ exists and

$$
J^{\prime}(x)=L(x)=-\int_{0}^{1} \frac{\left[(1-t)^{\alpha} f(t)\right]^{\prime}}{t-x} d t .
$$


Consequently, we have

$$
\begin{aligned}
Q^{\prime}(x) & =\int_{0}^{1} \frac{(\sqrt{1-t} / \sqrt{x}-\sqrt{t} / \sqrt{1-x})\left[(1-t)^{\alpha} f(t)\right]^{\prime}}{\sqrt{x} \sqrt{1-t}+\sqrt{t} \sqrt{1-x}} d t \\
& +\int_{0}^{1} \frac{\left[(1-t)^{\alpha} f(t)\right]^{\prime}}{t-x} d t \\
= & \int_{0}^{1} \frac{(\sqrt{1-t} / \sqrt{x}-\sqrt{t} / \sqrt{1-x})(\sqrt{x} \sqrt{1-t}-\sqrt{t} \sqrt{1-x})\left[(1-t)^{\alpha} f(t)\right]^{\prime}}{x-t} d t \\
& +\int_{0}^{1} \frac{\left[(1-t)^{\alpha} f(t)\right]^{\prime}}{t-x} d t \\
= & \frac{1}{\sqrt{x} \sqrt{1-x}} \int_{0}^{1} \frac{\sqrt{t} \sqrt{1-t}\left[(1-t)^{\alpha} f(t)\right]^{\prime}}{t-x} d t .
\end{aligned}
$$

This completes the proof.

Let us now find the solution $\varphi$ of the ordinary Abel equation (2.3). First note that (2.3) is equivalent to

$$
\int_{0}^{x} \frac{\varphi(t)}{(x-t)^{\alpha}} d t=\frac{-Q(x)}{2 \pi(1-x)^{\alpha} \sin \pi \alpha}
$$

so that

$$
\varphi(x)=-\frac{1}{2 \pi^{2}} \frac{d}{d x} \int_{0}^{x} \frac{Q(t) d t}{(1-t)^{\alpha}(x-t)^{1-\alpha}} .
$$

Now integrate by parts and note that $Q(0)=0$ by (2.4). Consequently, we have

$$
\begin{aligned}
\varphi(x) & =\frac{-1}{2 \pi^{2} \alpha} \frac{d}{d x} \int_{0}^{x}(x-t)^{\alpha}\left[(1-t)^{-\alpha} Q(t)\right]^{\prime} d t \\
& =-\frac{1}{2 \pi^{2}} \int_{0}^{x} \frac{\left[(1-t)^{\alpha} Q(t)\right]^{\prime}}{(x-t)^{1-\alpha}} d t
\end{aligned}
$$

or, equivalently,

$$
\varphi(x)=-\frac{1}{2 \pi^{2}} \int_{0}^{x} \frac{P(t) d t}{(x-t)^{1-\alpha}(1-t)^{1+\alpha}},
$$

where $P(t)=\alpha Q(t)+(1-t) Q^{\prime}(t)$.

In order to express $P(t)$ in terms of the function $f$, we apply the lemma pertaining to the differentiability of $Q(x)$. Thus we find that

$$
\begin{aligned}
P(x)= & \alpha \sqrt{x} \sqrt{1-x} \int_{0}^{1} \frac{(1-t)^{\alpha} f(t) d t}{\sqrt{t} \sqrt{1-t}(t-x)} \\
& +\frac{\sqrt{1-x}}{\sqrt{x}} \int_{0}^{1} \frac{\sqrt{t} \sqrt{1-t}\left[(1-t)^{\alpha} f(t)\right]^{\prime} d t}{t-x}
\end{aligned}
$$


and, after performing the indicated differentiation of the quantity in the integrand of the second integral, it follows that

$$
P(x)=\frac{\sqrt{1-x}}{\sqrt{x}}\left(\int_{0}^{1} \frac{\sqrt{t}(1-t)^{\alpha+1 / 2} f^{\prime}(t) d t}{t-x}-\alpha C\right)
$$

where

$$
C=\int_{0}^{1} t^{-1 / 2}(1-t)^{\alpha-1 / 2} f(t) d t
$$

Hence, by virtue of (2.5) and (2.6), we have a formula for the function $\varphi$. Therefore our solution of (1.1) is given by (1.2) with $\varphi$ as in (2.5).

We may now formulate the following theorem.

TheOREM. For $0<\alpha<1$ and $f(x) \in D(H)$, let

$$
F(x)=\int_{0}^{1} \frac{\sqrt{t}(1-t)^{\alpha+1 / 2} f^{\prime}(t) d t}{x-t} \text { and } C=\int_{0}^{1} t^{-1 / 2}(1-t)^{\alpha-1 / 2} f(t) d t \text {. }
$$

Then the sectionally holomorphic function

$$
\Phi(z)=\left(\int_{0}^{1}(t-z)^{-\alpha} \varphi(t) d t\right)^{1 / \alpha}, \quad z \notin[0,1]
$$

where

$$
\varphi(x)=\frac{1}{2 \pi^{2}} \int_{0}^{x} \frac{F(t) d t}{\sqrt{t}(1-t)^{\alpha+1 / 2}(x-t)^{1-\alpha}}+\frac{\alpha C \Gamma(\alpha) x^{\alpha-1 / 2}}{2 \pi^{3 / 2} \Gamma(\alpha+1 / 2) \sqrt{1-x}},
$$

solves the nonlinear boundary problem $\left(\Phi^{+}(x)\right)^{\alpha}+\left(\Phi^{-}(x)\right)^{\alpha}=f(x), 0<x<1$.

EXAMPLE. Given that $\alpha=\frac{3}{4}$ and $f(x)=32 x^{1 / 4}(1-x)+8 \sqrt{2}(1-x)^{1 / 4}(4 x-3)$ +1 , find $\Phi(z)$ satisfying (1.1).

Solution. The reader will not encounter any difficulty in verifying that

$$
\begin{gathered}
\int_{0}^{1} \frac{\sqrt{t} \sqrt{1-t}}{t-x} d t=\frac{\pi}{2}(1-2 x), \\
\int_{0}^{1}\left(\frac{1-t}{t}\right)^{1 / 4} \frac{d t}{t-x}=\pi\left(\frac{1-x}{x}\right)^{1 / 4}-\pi \sqrt{2}
\end{gathered}
$$

for $0<x<1$. An excellent method for evaluating these integrals is found in Levinson's paper [3]. These results will be used to evaluate the singular integral

$$
F(x)=\int_{0}^{1} \frac{\sqrt{t}(1-t)^{5 / 4} f^{\prime}(t)}{x-t} d t .
$$


We find that

$$
\begin{aligned}
F(x)= & \int_{0}^{1}\left[8\left(\frac{1-t}{t}\right)^{1 / 4}\left(1-6 t+5 t^{2}\right)+2 \sqrt{2} \sqrt{t} \sqrt{1-t}(19-20 t)\right] \frac{d t}{x-t} \\
= & \int_{0}^{1}\left[8\left(\frac{1-t}{t}\right)^{1 / 4}\left(6-5 x-5 t-\frac{5 x^{2}-6 x+1}{t-x}\right)\right. \\
& \left.+2 \sqrt{2} \sqrt{t} \sqrt{1-t}\left(20-\frac{19-20 x}{t-x}\right)\right] d t \\
= & \frac{9 \pi \sqrt{2}}{4}-8 \pi\left(1-6 x+5 x^{2}\right)\left(\frac{1-x}{x}\right)^{1 / 4} .
\end{aligned}
$$

Also the constant $C$ defined by $(2.7)$ is

$$
C=\int_{0}^{1} t^{-1 / 2}(1-t)^{1 / 4} f(t) d t=-3 \pi \sqrt{2}+\frac{\Gamma^{2}(1 / 4)}{3 \sqrt{2 \pi}},
$$

and hence the formula for $\varphi(x)$ in the theorem implies $\varphi(x)=\sqrt{2}(5 x-4)+$ $x^{1 / 4} /(2 \pi \sqrt{1-x})$. Thus it follows from (1.2) that

$$
\begin{aligned}
\Phi(z)=\left[4 \sqrt{2}(1-z)^{1 / 4}(4 z-3)+16 \sqrt{2}(-z)^{1 / 4}(1-z)\right. & \\
& \left.+\int_{0}^{1} \frac{t^{1 / 4} d t}{2 \pi \sqrt{1-t}(t-z)^{3 / 4}}\right]^{4 / 3} .
\end{aligned}
$$

The fact that this function solves (1.1) can be verified by direct substitution. For instance,

$$
\left(\Phi^{+}(x)\right)^{3 / 4}+\left(\Phi^{-}(x)\right)^{3 / 4}=32 x^{1 / 4}(1-x)+8 \sqrt{2}(1-x)^{1 / 4}(4 x-3)+K,
$$

where

$$
K=\frac{-\sqrt{2}}{2 \pi} \int_{0}^{x} \frac{t^{1 / 4} d t}{\sqrt{1-t}(x-t)^{3 / 4}}+\frac{1}{\pi} \int_{x}^{1} \frac{t^{1 / 4} d t}{\sqrt{1-t}(t-x)^{3 / 4}}
$$

We now show that $K=1$ by using the following formulae for the hypergeometric function [4]:

$$
\left\{\begin{array}{l}
F(a, b ; c ; x)=(1-x)^{c-a-b} F(c-a, c-b ; c ; x), \\
F(a, b ; c ; x)=\frac{\Gamma(c) \Gamma(c-a-b)}{\Gamma(c-a) \Gamma(c-b)} F(a, b ; a+b-c+1 ; 1-x) \\
\quad+(1-x)^{c-a-b} \frac{\Gamma(c) \Gamma(a+b-c)}{\Gamma(a) \Gamma(b)} F(c-a, c-b ; c-a-b+1 ; 1-x) .
\end{array}\right.
$$


Now it is easy to see by virtue of a variable change from $t$ to $u$ that

$$
\begin{aligned}
K= & -\frac{\sqrt{2 x}}{2 \pi} \int_{0}^{1} u^{1 / 4}(1-u)^{-3 / 4}(1-x u)^{-1 / 2} d u \\
& +\frac{(1-x)^{-1 / 4}}{\pi} \int_{0}^{1} \frac{[1-(1-x) u]^{1 / 4}}{\sqrt{u}(1-u)^{3 / 4}} d u \\
= & \frac{\sqrt{2} \Gamma^{2}(1 / 4)}{4 \pi^{3 / 2}}\left[-\sqrt{x} F\left(\frac{1}{2}, \frac{5}{4} ; \frac{3}{2} ; x\right)+2(1-x)^{-1 / 4} F\left(-\frac{1}{4}, \frac{1}{2} ; \frac{3}{4} ; 1-x\right)\right] \\
= & \frac{\sqrt{2} \Gamma^{2}(1 / 4)}{4 \pi^{3 / 2}}\left[-\frac{\sqrt{\pi} \Gamma(-1 / 4)}{2 \Gamma(1 / 4)}-2 \sqrt{x}(1-x)^{-1 / 4} F\left(1, \frac{1}{4} ; \frac{3}{4} ; 1-x\right)\right. \\
= & 1+\frac{\sqrt{2} \Gamma^{2}(1 / 4)}{2 \pi^{3 / 2}}(1-x)^{-1 / 4}\left[F\left(-\frac{1}{4} ; \frac{1}{2} ; \frac{3}{4} ; 1-x\right)\right.
\end{aligned}
$$

But the quantity within the brackets vanishes by virtue of the first formula in (2.8); hence $K=1$ and the solution is verified.

3. A power type boundary problem with a variable coefficient. The boundary problem

$$
\left(\Phi^{+}(x)\right)^{\alpha}+G(x)\left(\Phi^{-}(x)\right)^{\alpha}=f(x) \text { on }(0,1) \text { with } 0<\alpha<1
$$

can be put in the form (1.1) by using the sectionally holomorphic function $X$ defined by

$$
X(z)=\exp \left(\frac{1}{2 \pi i \alpha} \int_{0}^{1} \frac{\log G(t)}{t-z} d t\right), \quad z \notin[0,1] .
$$

It is assumed that the Hölder continuous function $G(x) \neq 0$. The function $X(z)$ is a solution of the homogeneous boundary problem $\left(X^{+}(x)\right)^{\alpha}=G(x)\left(X^{-}(x)\right)^{\alpha}$, so that (3.1) becomes

$$
\left(\frac{\Phi^{+}(x)}{X^{+}(x)}\right)^{\alpha}+\left(\frac{\Phi^{-}(x)}{X^{-}(x)}\right)^{\alpha}=\frac{f(x)}{\left(X^{+}(x)\right)^{\alpha}},
$$

and the theory for (1.1) applies provided the function $f(x)\left(X^{+}(x)\right)^{-\alpha}$ belongs to the class $D(H)$.

\section{REFERENCES}

1. K. D. Sakalyuk, The generalized Abel integral equation, Dokl. Akad. Nauk SSSR 131 (1960), $748-751$.

2. N. I. Muskhelishvili, Singular integral equations. Boundary problems of function theory and their application to mathematical physics, Noordhoff, Groningen, 1953, pp. 42-43.

3. Norman Levinson, Simplified treatment of integrals of Cauchy type, the Hilbert problem and singular integral equations. Appendix: Poincaré-Bertrand formula, SIAM Rev. 7 (1965), 487-488.

4. M. Magnus, F. Oberhettinger and R. P. Soni, Formulas and theorems for the special functions of mathematical physics, Springer-Verlag, Berlin and New York, 1966, p. 47.

Department of Mathematics, Southwest Missouri State University, Springfield, Missouri 65804 - 0094 